\title{
Prenatal factors in the aetiology of testicular cancer: an epidemiological study of childhood testicular cancer deaths in Great Britain, 1953-73
}

\author{
A J SWERDLOW, ${ }^{1}$ C A STILLER, ${ }^{2}$ AND L M KINNIER WILSON ${ }^{3}$ \\ From the Oxford Regional Health Authority, ${ }^{1}$ Headington, Oxford, the Childhood Cancer Research Group, ${ }^{2}$ \\ Department of Paediatrics, Radcliffe Informary, Oxford, and the Cancer Epidemiology Research Unit, ${ }^{3}$ \\ Birmingham University, Birmingham, UK.
}

SUMMARY A case-control study is reported based on 87 deaths from testicular cancer that occurred in children in Great Britain 1953-73. Factors that significantly increased relative risk were tuberculosis of the mother during the index pregnancy and maternal epilepsy; factors that increased risk but not significantly were hyperemesis in the index pregnancy, a maternal history of stillbirths, and hernia and genitourinary defects in the child. Cryptorchidism was not studied. The available evidence suggests that prenatal determinants of testicular cancer in adults are also determinants of testicular cancer in childhood. The incidence and mortality from this disease are not increasing among children in Britain and other countries, whereas there is an increasing trend in young adults in several developed countries. Probably, therefore, the secular increase in the rates of young adult testicular cancer is due to factors that affect adults but not children, and hence are likely to be postnatal.

Testicular tumours in children are rare tumours about which little is known epidemiologically. The study of the aetiology of testicular cancer in childhood, however, has become of particular importance with the recent publication of studies ${ }^{1-3}$ suggesting that adult testicular cancer may have some prenatal determinants.

It is difficult to obtain information about prenatal factors for adult men, and when such information is obtained by questionnaire 20 or more years after birth, the quality of the data must be suspect. Investigation of risk factors for childhood testicular cancer largely overcomes these problems because of the far shorter period from birth to tumour. Because childhood testicular cancer shares with the adult disease some associations ${ }^{1-5}$ of probable prenatal aetiology, investigation of prenatal risk factors for the childhood cancer may illuminate the search for causes of the increasingly common ${ }^{36}$ adult malignancy.

Since 1953, the Oxford Survey of Childhood Cancer $(\mathrm{OSCC})^{7}$ has collected information on all deaths from cancer of children in Britain aged under 10; from 1961 the age range covered was expanded to all those dying under 16 years. Deaths are notified to OSCC by the Registrars General and therefore ascertainment of cases in the survey is probably virtually complete. Interviews of the mothers of children with cancer were completed for $67 \%$ of children in the survey who died during 1953-73; abstracts of antenatal clinical notes for the index pregnancy were completed for $60 \%$ of deaths from cancer in childhood for the same period. The OSCC thus provides an opportunity to examine the epidemiology of testicular cancers in childhood using a series of cases larger than any previously published except that from the United States by $\mathrm{Li}$ and Fraumeni. ${ }^{4}$

\section{Method}

The files of OSCC were searched for all children dying from testicular tumours during 1953-73; the controls chosen were all boys who died during the same period from malignant neoplasms other than of genital site or of teratoma histology. Relative risks of testicular cancer for the variables examined were estimated by calculating odds ratios for the unmatched case-control study; the significance of these odds ratios was tested by the Chi Square test with Yates's correction or, where small numbers necessitated, by Fisher's exact test. ${ }^{8}$ For the analysis 
of parental age, expected numbers of cases with parents in each age group were calculated from the controls, allowing for the distribution of year of birth in the cases since there are time trends in the ages of parents at the birth of their children; controls with Down's syndrome, whose incidence is known to be related to maternal age, were excluded from these calculations. For the factors $x$-ray examinations to the mother in pregnancy, history of miscarriages and stillbirths, and illnesses of the mother or child, negative responses were not specifically recorded in the survey, and therefore all patients not recorded as positive were treated as negative in the analysis. This assumption may lead in some instances to an underestimate of the true prevalence of positives in cases and controls, but since the control group were children dying contemporaneously from other malignant neoplasms, and the same questionnaire was used for both groups, there is no reason to believe that the underestimate would be biased between cases and controls and affect the estimation of the relative risks. Seasonality of birth month and month of diagnosis of cases were tested by Edwards's method.' Mean age-specific death rates from testicular cancer in Britain 1953-73 at each age under 10 years were calculated using as numerators the numbers of deaths recorded by OSCC, and as denominators, for each age, the mean of the populations of that age at the 1951,1961 , and 1971 censuses $^{10-12}$; mean age-specific death rates 1961-73 for ages 10-15 years were calculated using means of the 1961 and 1971 populations. Secular trends in age-specific incidence rates of testicular cancer in children in England and Wales 1962-74, and age-specific death rates 1953-78, were calculated from data published by the Registrar General ${ }^{12-16}$; rates were calculated for three-year periods (plus the four-year period 1971-4 for incidence, and the two-year period $1977-8$ for deaths) to give larger numbers to reduce the importance of random variation.

\section{Results}

From 1953 to 1973 in Britain there were notified to OSCC 87 deaths of boys from genital cancers, all of which were testicular cancers, 35 deaths from teratomas of non-genital sites, and 10128 deaths from other malignant neoplasms in boys. The distribution of age at death from testicular tumours

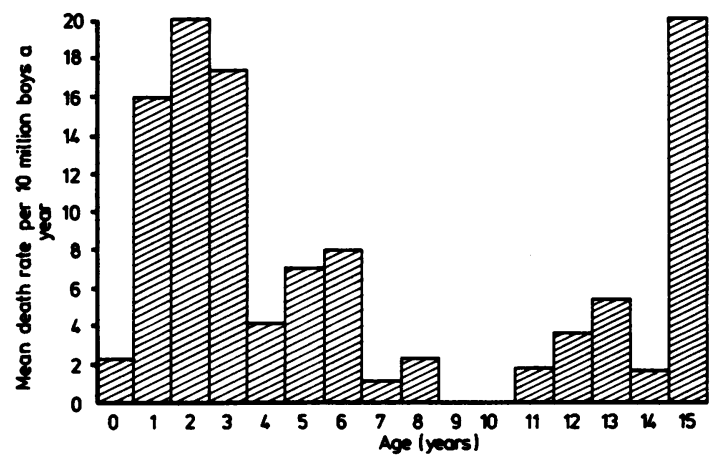

Fig 1 Mean age-specific childhood testicular death rates a year, Britain, ages 0-9 years 1953-73, and ages 10-15 years 1961-73.

Table 1 Demographic characteristics of parents

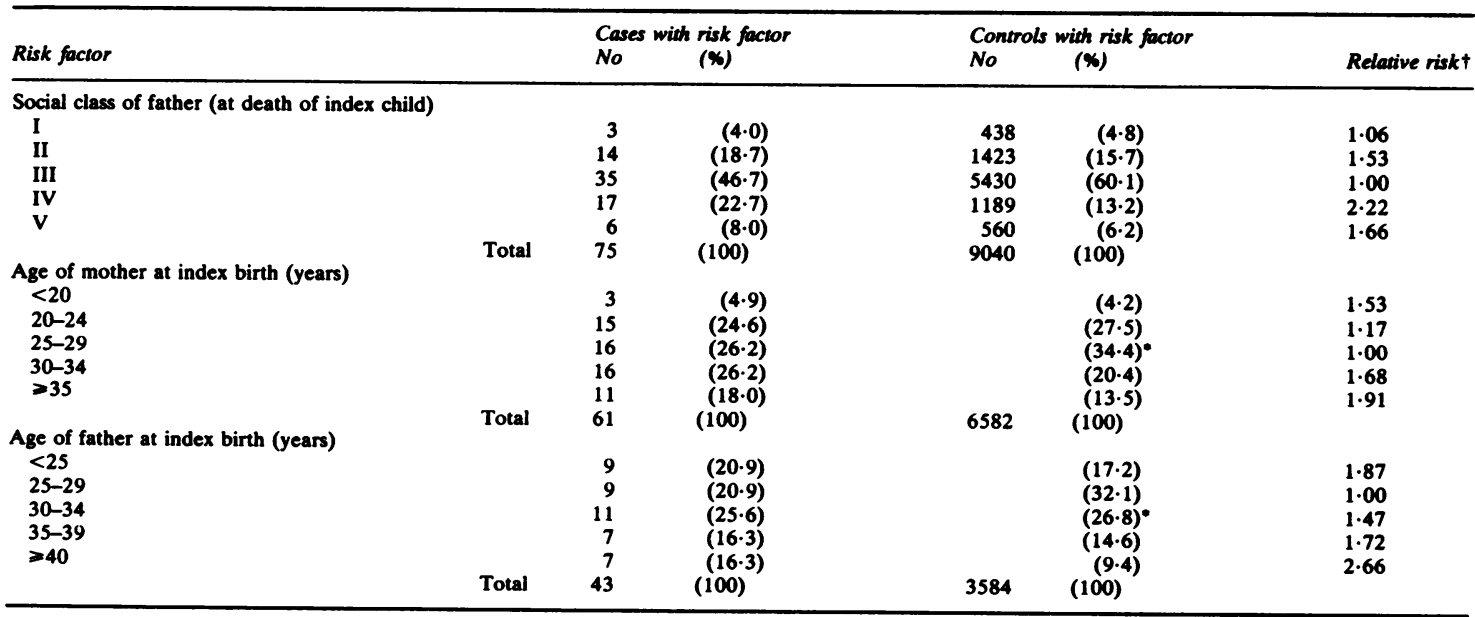

"Expected percentages (for method of derivation see text).

tFor none of the relative risks is $p<0.05$. 
(fig 1) showed a peak around age 2 years that included several histological types and a possible minor peak at age $5-6$ years when rhabdomyosarcoma was the commonest histology. There was a decrease from that age until 11 when an increase began, culminating in the large peak of mortality in young adults ${ }^{3,17}$; these adolescent tumours were largely teratomas.

There was no indication that incidence or mortality from testicular cancer in England and Wales (fig 2) have been rising in any childhood age group in recent years. Previous publications ${ }^{17}$ showed no rise from 1911 to 1969 in the death rate for the age group 0-14 years.

DEMOGRAPHIC FACTORS (table 1)

Risk of testicular cancer was not significantly related to social class, nor to age of the mother or father at the time of the patient's birth, although there was a non-significant rise in the relative risk to sons of older parents.

PREVIOUS OBSTETRIC HISTORY OF MOTHER (table 2)

Relative risk was raised for boys born to mothers who had had any stillbirths before the interview (relative risk $(R R)=1.96 ; p>0.05$ ), but miscarriages to the mother did not affect risk. There was no significant gradient or association of risk with sibship position.

MOTHER'S BLOOD GROUP, $\boldsymbol{X}$ - RAY EXAMINATIONS, AND ILLNESSES DURING PREGNANCY (table 3)

Blood group was known for only 36 of the mothers of patients with testicular cancer and was not significantly related to risk of testicular cancer in the

Table 2 Previous obstetric history of mother

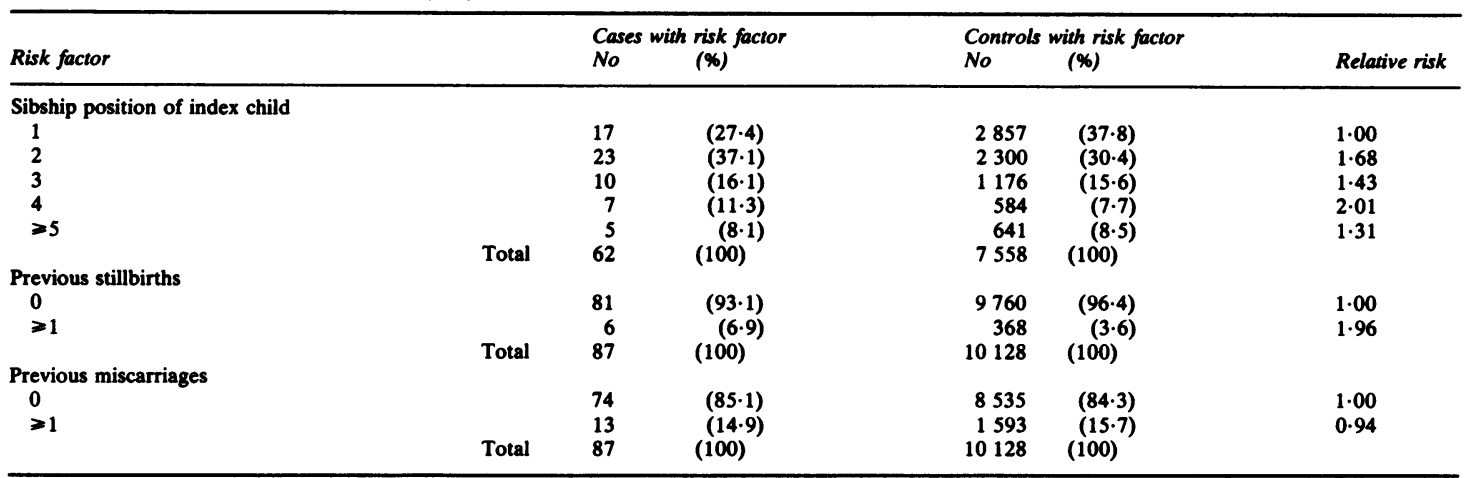

"For none of the relative risks is $\mathrm{p}<0.05$.

Table 3 Mother's blood group, x-ray examinations, and illnesses during pregnancy

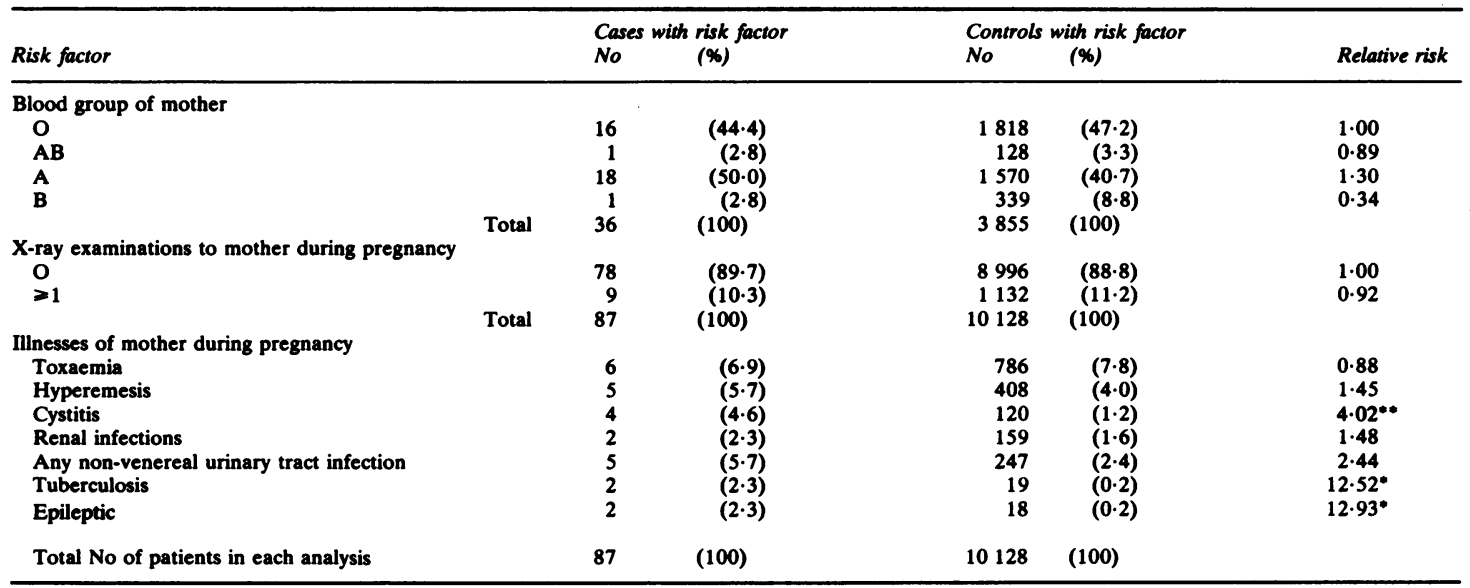

Probability p < 0.02; by Fisher's exact test.

$\bullet p<0.005$. 

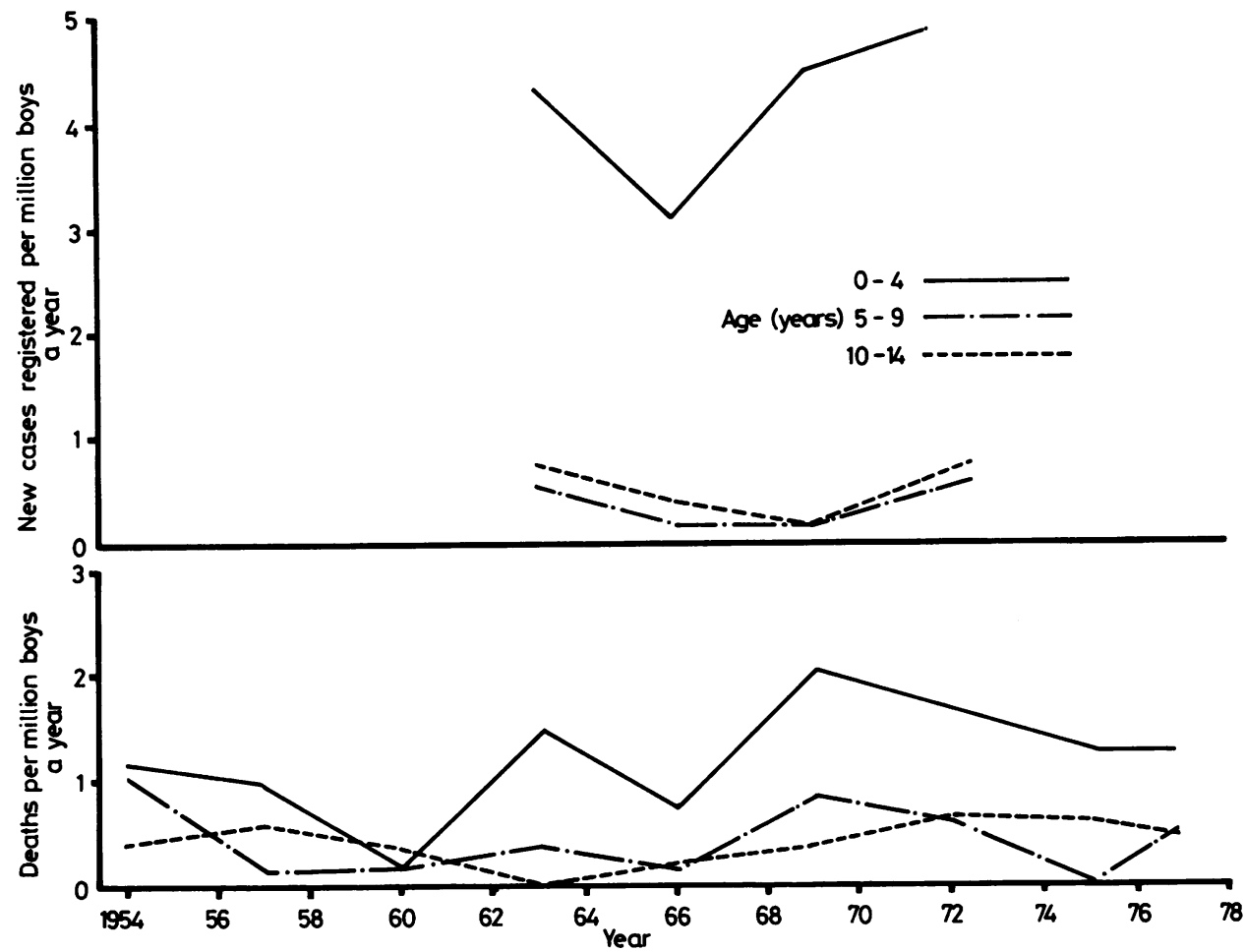

Fig 2 Childhood testicular cancer, England and Wales. Age-specific incidence rates for three-year periods $1962-74^{13} 16$ and age-specific death rates for three-year periods $1953-78 .^{15} 16$

son. $X$-ray examinations of the mother in pregnancy did not affect risk relative to that for other childhood cancers. Significantly raised risks of testicular cancer were present for tuberculosis of the mother during pregnancy $(R R=12 \cdot 5, p=0.014)$ and for epileptic mothers $(R R=12.9, p=0.012)$. Neither of the case-mothers with tuberculosis in pregnancy (one with tuberculosis of the kidney, the other with tuberculosis of the spine) had known drug treatment during the index pregnancy; both of the epileptic case-mothers had taken phenobarbitone during the index pregnancy, and one had also taken phenytoin. Significantly raised risk was also present for sons of mothers with cystitis in pregnancy. The raised risk, however, was less and non-significant for any urinary tract infection recorded in pregnancy, which were in practice cystitis or renal infections; no cases or controls were recorded with infections of other parts of the urinary tract, venereal infections were not included in the analysis, and bacteriuria per se was not recorded in the survey.
ILLNESSES OF THE CHILD, SEASONALITY OF BIRTH. AND DIAGNOSIS DATES

Two cases were recorded with genitourinary defects, of which one was a case of hypospadias and one of hydrocoele, but cryptorchidism was not recorded in the survey; this gave a relative risk of testicular cancer of $2.99(p=0.15)$ for boys with genitourinary defects. Congenital hernia was recorded only in the later years of the survey, and childhood hernias were recorded only if they had become strangulated or had been operated on; two cases had inguinal hernias recorded, a relative risk of $2.05(p>0.05)$. No significant seasonality was present for month of birth (number of cases $(n)=84, x_{2}^{2}=0.40$ ) or month of diagnosis $\left(n=74, x_{2}^{2}=1 \cdot 89\right)$.

\section{Discussion}

The study shows a peak of childhood testicular cancer mortality at age 2 years and a sharp rise at age 15 , very similar to the age mortality pattern in the United 
States. In England and Wales $1962-74$ as in Denmark 1943-62 ${ }^{17}$ and Connecticut 1935-76 there has not been any substantial secular increase in the incidence rates of childhood testicular cancer. Mortality rates in England and Wales 1911-78 and the United States 1931-76 41718 have also shown no secular increase, and probably reflect unchanged incidence over these periods. Japan is an exception to this pattern, with a rapid increase in childhood testicular cancer mortality $1947-70 .^{19}$ In contrast to the general secular pattern in children there has been a substantial rise in incidence and mortality from testicular cancer in young adults in several developed countries in recent years ${ }^{3817}$; this implies that if prenatal factors are aetiological for adult testicular cancer, they are either different prenatal factors from those aetiological for the childhood cancer or they are not the cause of the increasing secular trend. The evidence so far available is against the former explanation: testicular cancer in children has been found to be associated with cryptorchidism, hernia, and genitourinary defects, ${ }^{4}$ all of which probably have prenatal causes and all of which may well be associated with the adult cancer. ${ }^{15}$ Associations which were not significant, have also been found between adult testicular cancer and hyperemesis of the mother during pregnancy ${ }^{1}$ and between adult testicular cancer and intake of hormones by the mother in pregnancy, ${ }^{1-3}$ although the latter may not be a strong risk factor ${ }^{3}$ and is unlikley to account for the rising incidence of testicular cancer in developed countries. Data on hormone intake by the mother in pregnancy were not generally available for the present study, but the relative risk of childhood testicular cancer was slightly raised for hyperemesis of the mother during the index pregnancy. Overall, although the available evidence is not conclusive, it is consistently in the direction of childhood tumours sharing prenatal determinants of the adult disease.

It seems reasonable to suppose that any socioeconomic factor responsible for the secular increase in incidence of adult testicular cancer in developed countries also would be responsible for the strong association of the cancer with high social class $^{314}{ }^{20}$ and for the higher incidence rates in more developed than less developed countries ${ }^{21}$; it would be consistent with this being a postnatal factor if childhood testicular cancer showed no strong secular increase in incidence, no excess risk in high social classes, nor a higher incidence in developed countries. In the present study there was no evidence of higher rates of childhood testicular cancer in high social classes; there are not at present available sufficient population-years of data for different countries to judge the extent of international variation in the incidence rates of childhood testicular cancer, but when such data become available they will provide a test of the hypothesis.

The study showed a significantly raised risk of testicular cancer for two prenatal factors, tuberculosis and epilepsy of the mother, which have not previously been reported. The raised risk for sons of epileptic mothers is particularly noteworthy in view of previous findings of excess risk of congenital defects in offspring of epileptic mothers. ${ }^{22}$ The possible association of raised risk with urinary tract infections of the mother is of interest because of reports of increased perinatal mortality and low birthweight in those born to mothers with bacteriuria in pregnancy. ${ }^{23}$ The significance of these findings of risk from maternal illnesses in pregnancy should be interpreted with caution because the diseases found to give statistically significant risks were not chosen by prior hypothesis. The significance levels could not be satisfactorily corrected for multiple comparisons because it is not possible to delineate definitively what would count as "a comparison," given the undefinable number of diseases and combinations of diseases that could occur, and also because one cannot assume that all of these diseases occur independently. The risk factors need to be re-examined in future studies.

Several factors showed no significant association with risk in the present study, but moderate associations might easily not have shown up because the study was not large, data for some variables (blood group of the mother, age of the father, and hernia in the child) were only collected for part of the survey period, and the controls used would have tended to obscure any small increases in risk for the variables $x$-ray examinations in pregnancy and social class, which are known to have associations with childhood cancer in general. ${ }^{24}{ }^{25}$

The non-significantly raised risk of testicular cancer found for sons of mothers with previous stillbirths accords with a recent report of an excess of stillbirths to the mothers of patients with germ-cell tumour in childhood. ${ }^{26}$ Studies of sibling position ${ }^{127}$ and of mother's and father's age at the time of delivery of adults with testicular cancer have, like the present study, not reported significant relationship with risk. No studies of the blood group of mothers of patients with testicular cancer have previously been published; a study of the blood group of adult patients with testicular cancer ${ }^{27}$ did not show any strong relationship of blood group to risk. The increasing evidence that testicular cancer has some prenatal risk factors, possibly preventable, makes it important that larger studies of such factors should be undertaken. It would be extremely difficult to carry out a larger study of childhood testicular cancer than the present one. Because of the much higher 
incidence of testicular cancer in young adults than in children, the most useful practical approach to further investigation of the prenatal determinants of testicular cancer would be a study of the obstetric case notes of young men with testicular tumours, together with those of a suitable group of controls.

We thank Dr G J Draper for advice and helpful criticism and Mrs S Jones and Mrs N Cross for typing the manuscript.

\section{References}

${ }^{1}$ Henderson BE, Benton B, Jing J, Yu MC, Pike MC. Risk factors for cancer of the testis in young men. Int $J$ Cancer 1979; 23: 598-602.

${ }^{2}$ Loughlin JE, Robboy SJ, Morrison AS. Risk factors for cancer of the testis. $N$ Engl J Med 1980; 303: 112-3.

${ }^{3}$ Schottenfeld D, Warshauer ME, Sherlock S, Zauber AG, Leder M, Payne R. The epidemiology of testicular cancer in young adults. Am J Epidemiol 1980; 112: 232-46.

'Li FP, Fraumeni JF Jr. Testicular cancers in children: epidemiologic characteristics. J Natl Cancer Inst 1972; 48: 1575-82.

${ }^{5}$ Morrison AS. Cryptorchidism, hernia and cancer of the testis. J Natl Cancer Inst 1976; 56: 731-3.

- Anonymous. An epidemic of testicular cancer? Lancet 1968; ii: $164-5$.

${ }^{7}$ Stewart A, Webb J, Hewitt D. A survey of childhood malignancies. Br Med J 1958; i: 1495-1508.

Armitage P. Statistical methods in medical research. Oxford: Blackwell Scientific Publications, 1971.

${ }^{9}$ Edwards JH. The recognition and estimation of cyclic trends. Ann Hum Genet, 1961; 25: 83-6.

${ }^{10}$ General Register Office. Census 1951, England and Wales. General tables. London: HMSO, 1956.

1 General Register Office for Scotland. Census 1951. Vol III, general volume. Edinburgh: HMSO, 1954.

${ }^{12}$ General Register Office/Office of Population Censuses and Surveys. Censuses 1961, 1971, Great Britain. Summary tables. London: HMSO, 1966, 1973.
${ }^{13}$ General Register Office/Office of Population Censuses and Surveys. Registrar General's statistical review of England and Wales. Supplement on cancer. For 1962-4, 1965, 1966-7, 1968-70. London: HMSO, 1968, 1970, $1972,1975$.

${ }^{14}$ Office of Population Censuses and Surveys. Cancer statistics registrations. England and Wales, 1971, 1972-3, 1974. Series MB1 Nos 1, 2, 4. London: HMSO, 1979-80.

${ }^{15}$ General Register Office/Office of Population Censuses and Surveys. Registrar General's statistical review of England and Wales for the years 1953-73. Part I(A) tables, medical. London: HMSO, 1954-75.

${ }^{16}$ Office of Population Censuses and Surveys. Mortality statistics cause. England and Wales 1974-8. Series DH2 Nos 1-5. London: HMSO, 1977-80.

${ }^{17}$ Petersen GR, Lee JAH. Secular trends of malignant tumors of the testis in white men. J Natl Cancer Inst 1972; 49: 339-54.

${ }^{18}$ Grumet RF, MacMahon B. Trends in mortality from neoplasms of the testis. Cancer 1958; 11: 790-7.

${ }^{19}$ Lee JAH, Hitosugi M, Petersen GR. Rise in mortality from tumors of the testis in Japan, 1947-70. J Natl Cancer Inst 1973; 51: 1485-90.

${ }^{20}$ Office of Population Censuses and Surveys. Occupational mortality. The Registrar General's decennial supplement for England and Wales, 1970-2. Series DS No 1. London: HMSO, 1978.

${ }^{21}$ Waterhouse J, Muir C, Correa P, Powell J, eds. Cancer incidence in five continents. Vol III. Lyon: International Agency for Research on Cancer, 1976.

${ }^{22}$ Fedrick J. Epilepsy and pregnancy: a report from the Oxford Record Linkage Study. Br Med J 1973; ii: 442-8.

${ }^{23}$ Zinner SH. Bacteriuria and babies revisted. $N$ Engl $J$ Med 1979; 300: 853-5.

${ }^{24}$ Bithell JF, Stewart AM. Pre-natal irradiation and childhood malignancy: a review of British data from the Oxford Survey. Br J Cancer 1975; 31: 271-87.

${ }^{25}$ Adelstein AM, White GC. Causes of children's deaths analysed by social class (1959-63). In: Office of Population Censuses and Surveys. Child health: $A$ collection of studies. Studies on medical and population subjects No 31. London: HMSO, 1976; $23-40$.

${ }^{26}$ Birch JM, Marsden HB, Swindell R. The epidemiology of germ-cell tumours of childhood. Br J Cancer 1979; 40: 815.

${ }^{27}$ Morrison AS. Some social and medical characteristics of army men with testicular cancer. Am J Epidemiol 1976; 104: $511-6$. 